\title{
PHRAGMEN-LINDELOF THEOREMS FOR SOME NONLINEAR ELLIPTIC PARTIAL DIFFERENTIAL EQUATIONS ${ }^{1}$
}

\author{
BY JOSEPH J. ROSEMAN \\ Communicated by E. Isaacson, May 26, 1972
}

The term Phragmen-Lindelof theorem, when applied to an elliptic partial differential equation, refers here to a theorem of the following type:

One considers the set of all solutions of the differential equation in a semi-infinite cylinder, which satisfies homogeneous Dirichlet data on the long sides of the cylinder and certain a priori bounds throughout the cylinder. It is then concluded that the solution, or some integral norm of the solution, decays exponentially with distance from the face of the cylinder. Results of this type are given in [1] for various linear elliptic partial differential equations. ${ }^{2}$ In the mathematical theory of elasticity, Saint-Venant's Principle may be regarded as a particular example of a Phragmen-Lindelof theorem (cf. [4], [5], [6]).

In this paper, we consider nonlinear elliptic partial differential equations in two independent variables in the strip $R:[(x, y) / 0 \leqq x, 0 \leqq y \leqq h]$ of the form:

$$
\left(\frac{\partial^{2}}{\partial x^{2}}+\frac{\partial^{2}}{\partial y^{2}}\right)^{m} u=\sum_{0 \leqq j \leqq m} P_{|j|}(u) \partial^{|j|} u \partial^{|2 m-j|} u
$$

where $\partial^{|j|}$ represents a partial derivative operator of the form $(\partial / \partial x)^{r}(\partial / \partial y)^{j-r}$ and, for every $j$, the summation includes all operators of this form. $\left(\partial^{0} u=u\right)$.

For such a system, it is shown that

THEOREM 1. Consider a differential equation of type (1) in $R$, with $P_{|j|}(u) \in C^{m+2}[-\varepsilon, \varepsilon]$. Let $u$ be a solution of the equation, which satisfies homogeneous Dirichlet boundary data at $y=0$ and $y=h$ and such that, throughout $R$,

$$
\begin{aligned}
|u| & <\varepsilon<1, \\
\left|\partial^{|j|} u\right| & <1 / a^{j}, \quad j=1,2, \ldots, m .
\end{aligned}
$$

AMS (MOS) subject classifications (1970). Primary 35B05, 35B40, 35B45, 35J30, 35J60; Secondary $73 \mathrm{C} 10,31 \mathrm{~B} 30$.

Key words and phrases. Phragmen-Lindelof, elliptic, exponential decay, a priori estimates.

${ }^{1}$ Research supported by NSF-GP-27960.

${ }^{2}$ Other types of P-L theorems are given in [2] and [3]. 
Then, if $\varepsilon$ and $h / a$ are sufficiently small, $u$ and the first $m-1$ derivatives of $u$ decay exponentially as $x \rightarrow+\infty$ in $R$. The required a priori upper bounds for $\varepsilon$ and $h / a$, which determine the minimum rate of exponential decay, depend only upon the smoothness properties of the functions $P_{|j|}(u)$.

If $P_{|j|}(u)=0$, for all $j$, then (1) becomes a linear equation and it is only necessary to assume that $|u|<\varepsilon$ (not necessarily small) for the result to be true.

This theorem thus extends the known Phragmen-Lindelof theorems for the linear equation

$$
\left(\partial^{2} / \partial x^{2}+\partial^{2} / \partial y^{2}\right)^{m} u=0
$$

into a wide class of nonlinear elliptic equations.

It is further proved that

THEOREM 2. If equation (2b) is valid for $j=1,2, \ldots, 2 m$ and $P_{|j|}(u)$ $\in C^{2 m+2}[-\varepsilon, \varepsilon]$, then $u$ and the first $2 m-1$ derivatives of $u$ decay exponentially as $x \rightarrow+\infty$ in $R$, with the minimum rate of decay depending only upon the smoothness properties of the functions $P_{|j|}(u)$.

The techniques used to prove these theorems are similar to those employed in [6]. Beginning with the a priori estimates (2) valid throughout $R$, it is shown that, in the subdomain of $R$ in which $x>2 a$, there exists a constant $M$, which depends only upon the functions $P_{|j|}(u)$ such that

$$
|u|<\varepsilon M h / a, \quad x>2 a .
$$

The arguments can then be repeated to give

$$
|u|<\varepsilon(M h / a)^{n}, \quad x>2 n a,
$$

which implies exponential decay if $M h / a<1$.

Similar arguments are used for the derivatives of $u$.

Full proofs of these theorems will appear in the Journal of Mathematical Analysis and Applications.

\section{REFERENCES}

1. P. D. Lax, A Phragmén-Lindelöf theorem in harmonic analysis and its application to some questions in the theory of elliptic equations, Comm. Pure Appl. Math. 10 (1957), 361389. MR 20 \# 229.

2. J. B. Serrin, Jr., On the Phragmén-Lindelöf principle for elliptic differential equations, J. Rational Mech. Anal. 3 (1954), 395-413. MR 16, 42.

3. D. Gilbarg, The Phragmén-Lindelöf theorem for elliptic partial differential equations, 
J. Rational Mech. Anal. 1 (1952), 411-417. MR 14, 279.

4. R. A. Toupin, Saint-Venant's principle, Arch. Rational Mech. Anal. 18 (1965), 83-96. MR 30 \# 2725.

5. J. K. Knowles, On Saint-Venant's principle in the two-dimensional linear theory of elasticity, Arch. Rational Mech. Anal. 21 (1965), 1-22. MR 32 \# 4930.

6. J. J. Roseman, The principle of Saint-Venant in linear and non-linear plane elasticity, Arch. Rational Mech. Anal. 26 (1967), 142-162. MR 35 \#7630.

Department of Mathematics, Polytechnic Institute of Brooklyn, Brooklyn, New YORK 11201 